\title{
A field-deployable and handheld fluorometer for environmental water quality monitoring
}

\author{
Young-Ho Shin ${ }^{1}$, M. Teresa Gutierrez-Wing ${ }^{2}$ and Jin-Woo Choi ${ }^{1,3^{*}}$ (1)
}

\begin{abstract}
This work reports the development of a field-deployable and fully handheld fluorometer for environmental water monitoring. Our developed fluorimeter can detect both green algae and cyanobacteria (blue-green algae) while simultaneously differentiating and measuring two different species by selectively measuring chlorophyll $a$ fluorescence from green algae and phycocyanin fluorescence from blue-green algae. As a demonstration, chlorophyll $a$ and phycocyanin photopigments were estimated and differentiated. The system was also tested with an environmental water sample collected from a lake to validate the system functionality. The presented results suggest that our developed fluorimeter could be used as a portable water quality monitoring system for detection of different photopigment of algae.
\end{abstract}

Keywords: Green algae, Blue-green algae (cyanobacteria), Water quality monitoring, Fluorometer

\section{Introduction}

Distribution of phytoplankton groups provides important information about the environmental water quality because they are closely related with eutrophic status which often caused by the water contamination [1]. Rapid reproduction of algae in waterbody due to the inflow of nutrient containing nitrogen $(\mathrm{N})$ and phosphorus $(\mathrm{P})$ causes hypoxia that kills marine animals and disrupts the aquatic ecosystems. Some cyanobacteria (blue-green algae) species even produce cytotoxic substances that could pose problem for those of who get their drinking water from lakes or reservoirs contacting toxic blooms. Current widely used methods in phytoplankton detection and classification are microscopic examination [2], high-performance liquid chromatography (HPLC) [3], and bench-top flow-cytometry [4] which are time-consuming, expensive, and difficult to apply for the on-field detection. Fluorimetry is one of the widely used tools in detecting and analyzing different phytoplankton species

\footnotetext{
*Correspondence: choijw@lsu.edu

${ }^{1}$ School of Electrical Engineering and Computer Science, Louisiana State University, Baton Rouge, LA, USA

Full list of author information is available at the end of the article
}

by measuring the fluorescence signals emitted from the photopigments [5].

Previously, we have reported a fluorometer system in $[6,7]$ based on controlled samples. In this work, we demonstrate the functionality of our system at a local lake using environmental water samples collected on site. A hand-held fluorometer has three different wavelengths of excitation light emitting diodes (LEDs) to selectively stimulate the target phytoplankton species and corresponding fluorescence signal was measured with a photodetector. Electronic system including the microcontroller unit, liquid crystal display (LCD), and data storage unit are compactly assembled into a custom-made 3D-printed jig.

\section{Working concept}

Chlorophyll $a$ and phycocyanin are main photopigments in green and blue-green algae, respectively, and have different absorption and emission spectra that each pigment can be identified by selectively stimulating and detecting the corresponding fluorescence. Figure 1a shows the normalized excitation-emission matrices (EEMs) of chlorophyll $a$ and $\mathrm{C}$-phycocyanin pigments measured with a spectrofluorometer (Fluorolog ${ }^{\circledR}-3$, Horiba, Japan). The 

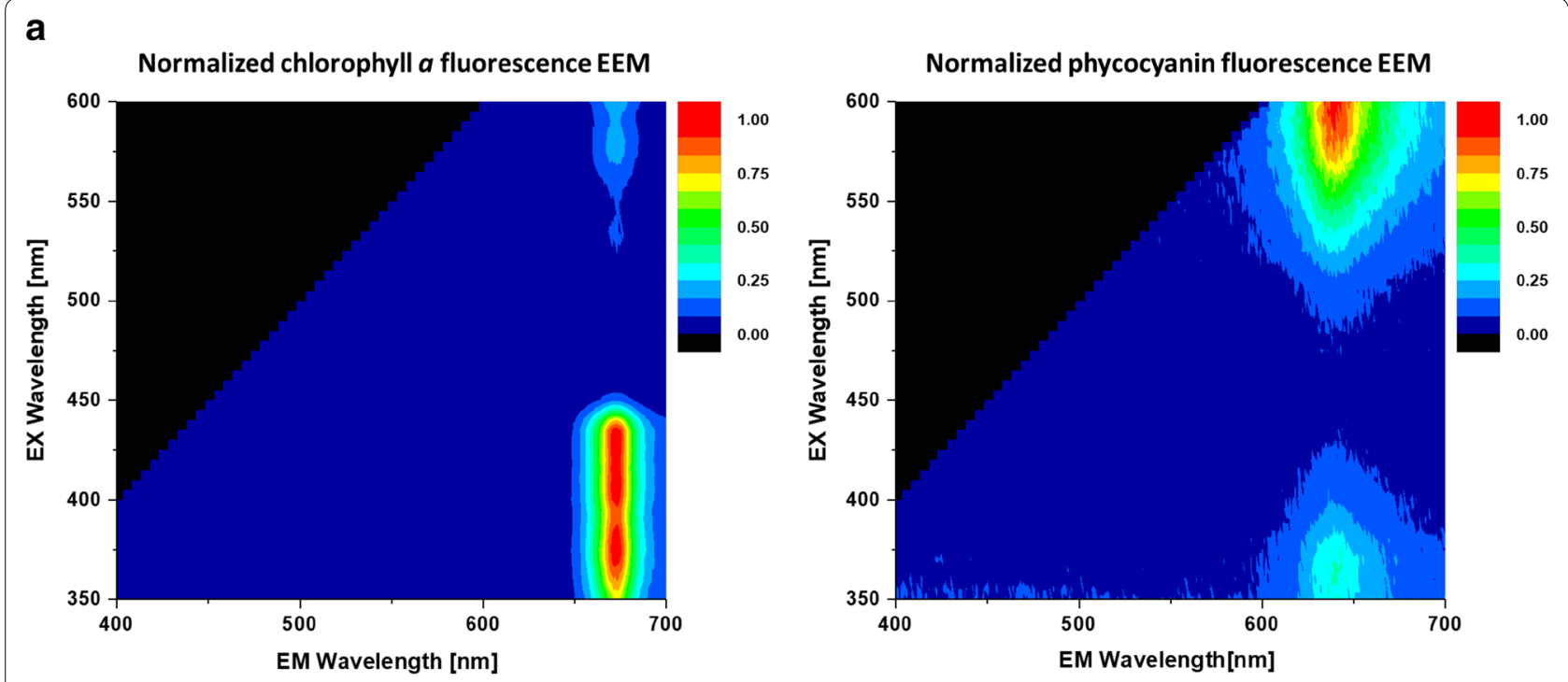

b

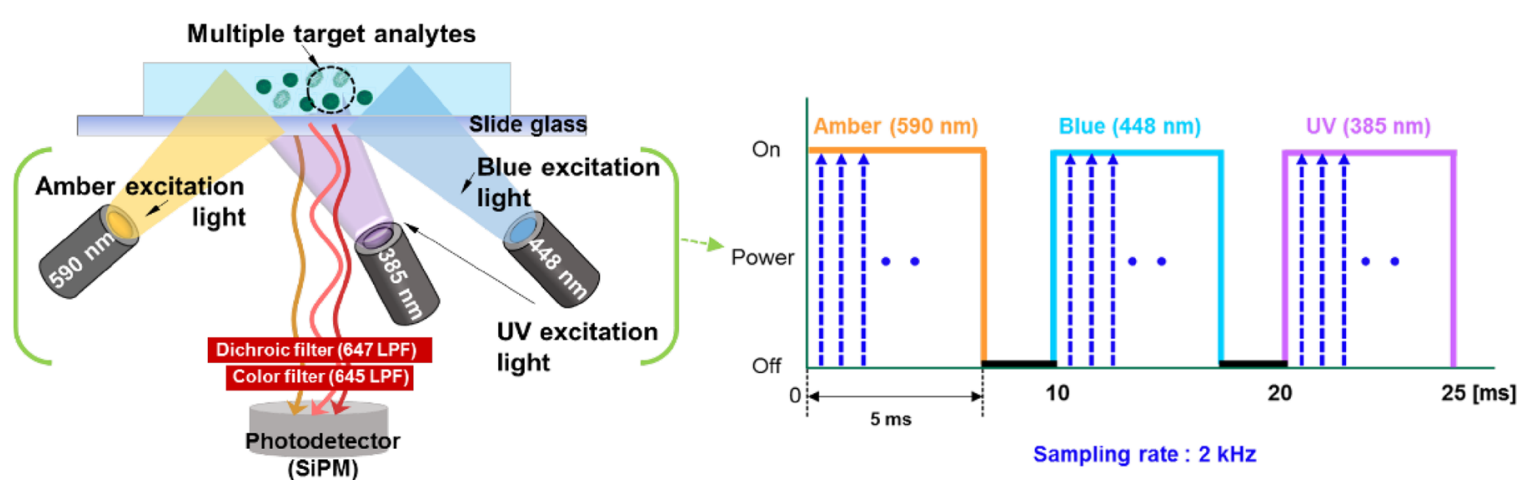

Fig. 1 Working principle of an LED based fluorescence detection: a normalized fluorescence excitation-emission matrices (EEMs) of chlorophyll a and phycocyanin pigments and $\mathbf{b}$ a schematic of the concept of an LED based fluorescence sensor platform

pseudo-colored (red being highest and blue being lowest) fluorescence intensity map is produced by scanning fluorescence signal over a range of excitation $(350-600 \mathrm{~nm})$ and emission wavelength $(400-700 \mathrm{~nm})$. The plot shows that chlorophyll $a$ absorbs the light most strongly in the blue region (360-440 $\mathrm{nm}$ ) and fluoresces in the red region $(660-680 \mathrm{~nm})$ while phycocyanin absorbs the light most strongly in the amber region (550-660 nm) and fluoresces in the red region $(630-650 \mathrm{~nm})$. Note that chlorophyll $a$ and phycocyanin also slightly absorb the light in the red and ultraviolet region, respectively.

A schematic illustration of our fluorometer system is shown in Fig. 1b. Three different wavelengths of excitation LEDs are used to selectively stimulate the distinct photopigment of target species. A blue LED (448 nm) is used to stimulate chlorophyll $a$ in microalgae while amber LED $(590 \mathrm{~nm})$ is used to stimulate phycocyanin in blue-green algae $[8,9]$. An ultraviolet (UV) LED $(360 \mathrm{~nm})$ is used to stimulate both chlorophyll $a$ and phycocyanin pigments. Three LEDs are implemented and sequentially turned on and off to selectively excite each pigment, and the corresponding fluorescence signals were measured with a highly sensitive silicon photomultiplier (SiPM). Two long-pass filters are mounted on top of the SiPM photosensor to block the excitation light while only allowing the longer wavelength fluorescence to pass. A $20 \mu \mathrm{l}$ aliquot of the sample solution is easily delivered with a disposable glass micro-vial.

\section{Materials and methods Sensor design}

A handheld fluorometer system, shown in Fig. 2, composed of an LCD screen, a data logging unit, custom designed circuit board, optical filters, and 


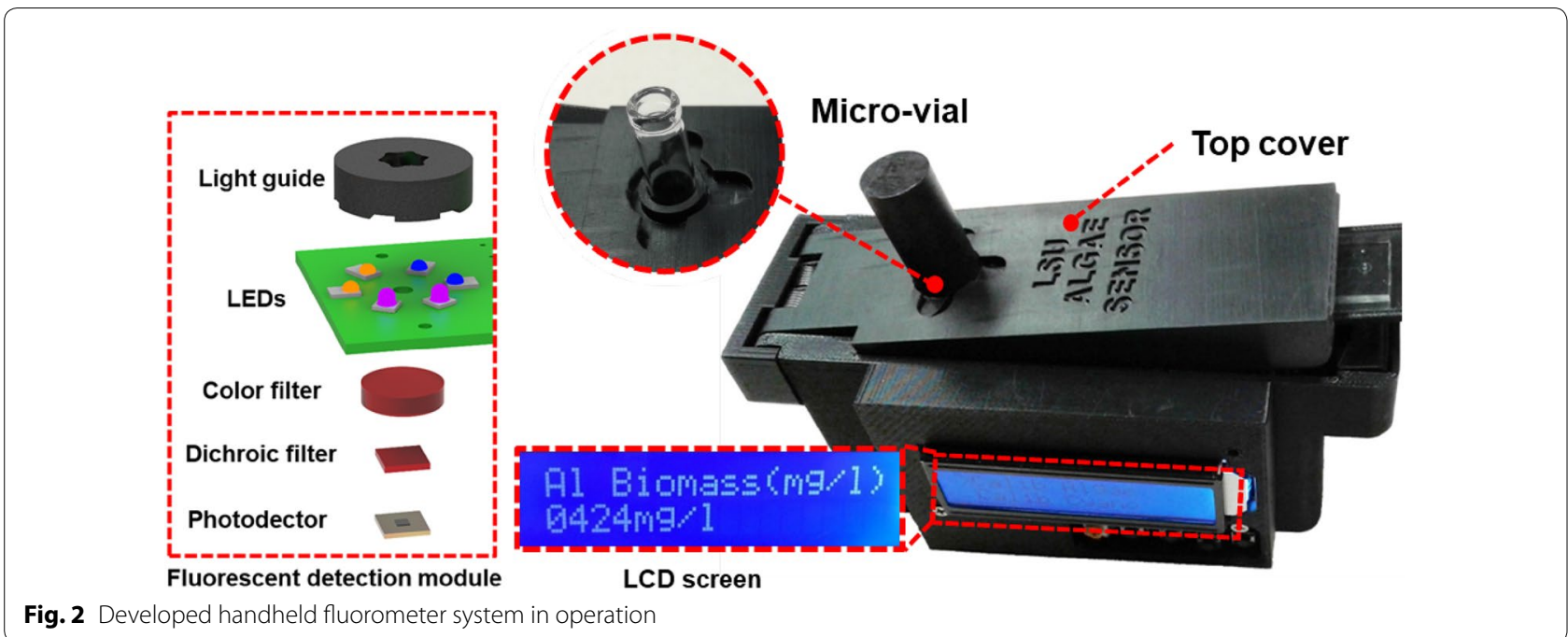

microcontroller, which are enclosed in a 3D-printed housing with a disposable glass micro-vial for a simple sample loading. A microcontroller module (Feather M0 Adalogger, Adafruit, USA) was utilized to control the excitation LEDs, conduct data acquisition, and display the results on the LCD screen. The fluorometer system was powered by a $9 \mathrm{~V}$ alkaline battery.

\section{Chlorophyll $a$ and phycocyanin fluorescence experiments} For the sample preparation, $1 \mathrm{mg}$ of chlorophyll $a$ powder (Sigma-Aldrich, MO, USA) was dissolved into a diethyl ether solvent (VWR, PA, USA) and $10.0 \mathrm{mg} \mathrm{ml}^{-1}$ of phycocyanin (Sigma-Aldrich, MO, USA) was mixed with deionized (DI) water. Sample stocks at a lower concentration were obtained by serially diluting the highest solution stocks for each pigment.

For measuring fluorescence signals of chlorophyll $a$ and phycocyanin, glass micro-vials were filled with different concentrations of $20 \mu \mathrm{l}$ sample volume. The measurements were conducted with three replicates of glass micro-vials for each concentration. An empty micro-vial was placed on the sensing aperture of the device and the sample solution was loaded into the vial. A light blocker cap was closed to block the ambient light noise. The LEDs were set to emit light intensity of $0.6 \mu \mathrm{E} \mathrm{m}^{-2} \mathrm{~s}^{-1}$ (or $122 \mathrm{~m} \mathrm{Wm}^{-2}$ ), $0.5 \mu \mathrm{E} \mathrm{m}^{-2} \mathrm{~s}^{-1}$ (or $109 \mathrm{~m} \mathrm{Wm}^{-2}$ ), and 0.35 $\mu \mathrm{E} \mathrm{m}^{-2} \mathrm{~s}^{-1}$ (or $134 \mathrm{~m} \mathrm{Wm}^{-2}$ ) for the amber, UV, and blue excitation LEDs, respectively.

\section{Results and discussion}

\section{Characterization of chlorophyll a and phycocyanin} fluorescence

In order to demonstrate the selective detection capability, different concentrations of chlorophyll $a$ and phycocyanin solutions were first tested to study fluorescence responsivity under different excitation LEDs for calibration shown in Fig. 3. The results show that fluorescence emission from chlorophyll $a$ mainly increased under the blue excitation LED, while the fluorescence emission from phycocyanin mainly increased under the amber excitation. Note that chlorophyll $a$ fluoresces under the UV LED as well, therefore this signal can be utilized to distinguish the fluorescence emission from the mixture of chlorophyll $a$ and phycocyanin pigments. This proves that the developed fluorometer can be used in detecting and differentiating the chlorophyll $a$ and phycocyanin pigments simultaneously. The data were used to selectively estimate two different species (green and blue-green algae) using the developed algorithm in our previous work [7]. The lower limit of detection for both chlorophyll $a$ and phycocyanin was set to $1 \mu \mathrm{g}^{-1}$ with $1 \mu \mathrm{g} \mathrm{l}^{-1}$ resolution. Based on the World Health Organization (WHO) water quality guidelines, Level 1 alert or low probability of acute health effect for the chlorophyll $a$ concentration and the phycocyanin are $<10 \mu \mathrm{g} \mathrm{l^{-1 }}$ and $<30 \mu \mathrm{g} \mathrm{l}^{-1}$, respectively, therefore our fluorometer suits for the water quality monitoring purpose $[10,11]$.

\section{Characterization of green algae and blue-green algae fluorescence}

In order to directly measure the chlorophyll $a$ and phycocyanin fluorescence from green algae (Chlorella vulgaris) and blue-green algae (Anabaena sp.), EEM of each species was measured with a spectrofluorometer shown in Fig. 4. It shows that the EEM patterns of green algae and blue-green algae agree well with that of chlorophyll $a$ and phycocyanin, respectively. Therefore, hand-held fluorometer can directly estimate the photopigment 

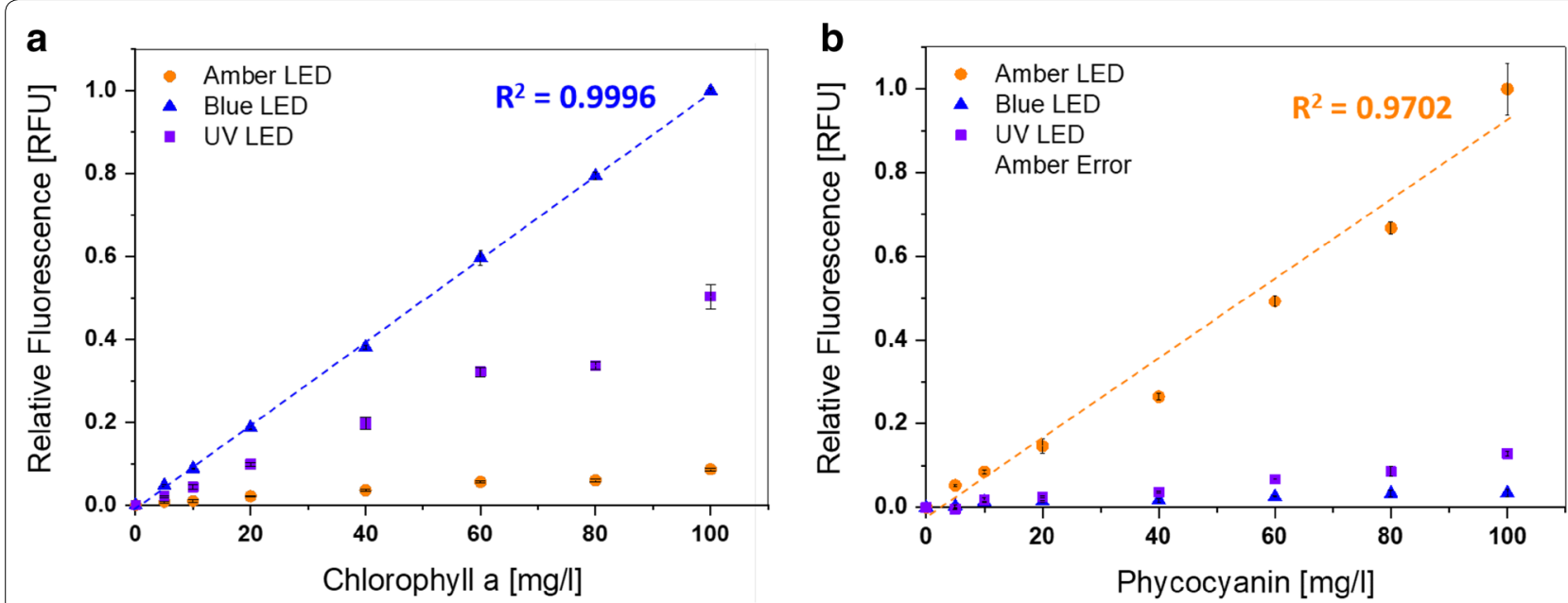

Fig. 3 Measured photocurrent of the fluorescence emission from the $\mathbf{a}$ chlorophyll $a$ and $\mathbf{b}$ phycocyanin using three different excitation LEDs. The error-bars represent the standard deviation of a data set $(n=3)$
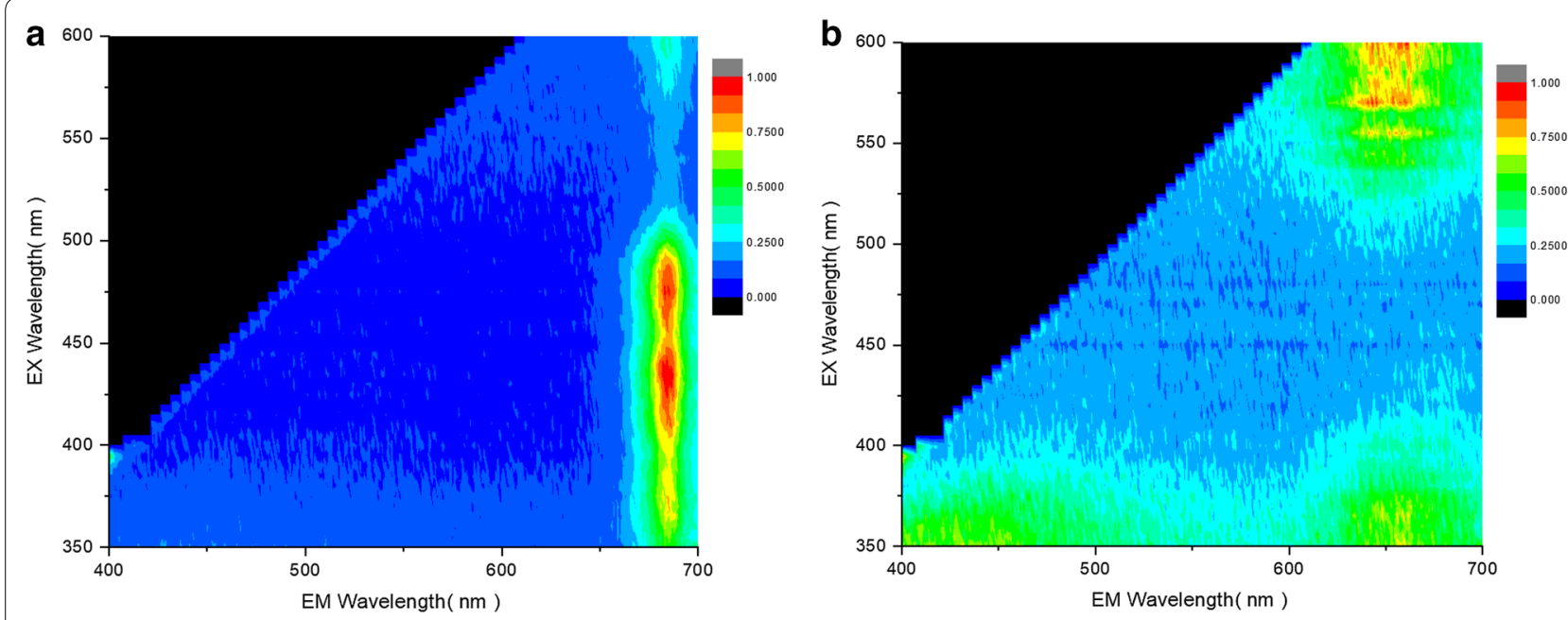

Fig. 4 Normalized fluorescence EEMs (excitation-emission matrices) of a green algae and $\mathbf{b}$ blue-green algae

concentration of green algae and blue-green algae after calibrating with pure photopigment. Note that the bluegreen algae sample shows UV fluorescence in 400$500 \mathrm{~nm}$ emission range due to the chromophoric (or colored) dissolved organic matter (CDOM) [12]. However, this does not interfere with fluorescence emission from green algae and blue-green algae fluorescence under blue LED (448 nm) and amber LED (590 nm) excitation.

\section{Identification of chlorophyll a and phycocyanin in a local lake water}

After calibration, the system was brought to a local lake and tested with environmental water samples collected at the scene. Figure 5 demonstrates on-site detection capabilities of our hand-held fluorometer system. First, lake water was delivered into a large glass vial with a syringe and later loaded into a disposable glass microvial for the measurement. The fluorometer displays and stores the data within $5 \mathrm{~s}$ of its operation and the user can always recalibrate the sensor if necessary.

Table 1 shows the measured results from the environmental samples. Three replicate measurements were relatively consistent for both chlorophyll $a$ and phycocyanin values. Based on our measurement result, a local lake has a moderate level of chlorophyll $a$ and low level of phycocyanin. The concentration of green algae in the lake was relatively higher due to several factors such as stagnant water and inflow of polluted local sewage water. 


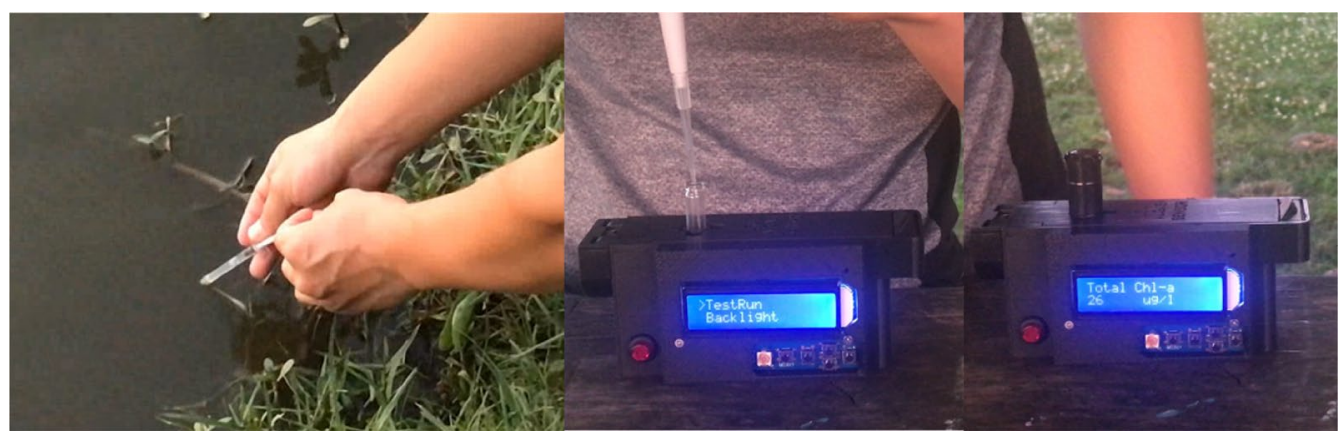

Fig. 5 In-situ measurement of chlorophyll $a$ and phycocyanin at a local lake using the developed handheld fluorometer

Table 1 The results of chlorophyll a and phycocyanin pigments estimated by measuring samples that are collected from the local lake

\begin{tabular}{|c|c|c|c|c|}
\hline Samples & $\begin{array}{l}\text { Test } 1 \\
\left(\mu \mathrm{gl}^{-1}\right)\end{array}$ & $\begin{array}{l}\text { Test } 2 \\
\left(\mu \mathrm{gl}^{-1}\right)\end{array}$ & $\begin{array}{l}\text { Test } 3 \\
\left(\mu \mathrm{gl}^{-1}\right)\end{array}$ & $\begin{array}{l}\text { Overall } \\
\left(\mu \mathrm{gl}^{-1}\right)\end{array}$ \\
\hline Chlorophyll a & 26 & 25 & 24 & $25.0 \pm 1.0$ \\
\hline Phycocyanin & 4 & 5 & 4 & $4.3 \pm 0.6$ \\
\hline
\end{tabular}

The results demonstrated that the developed fluorometer system with multi-excitation LEDs was able to selectively detect chlorophyll $a$ and phycocyanin in local lake water. The developed fluorometer can be potentially used as a fluorescence sensing platform for a portable biochemical analytic device.

\section{Conclusion}

In this work, we have developed a field-deployable handheld fluorimeter that could monitor the environmental water quality by detecting and distinguishing different photopigments of phytoplankton species. Green algae were detected by the chlorophyll a fluorescence under the blue LED excitation and blue-green algae were detected by the phycocyanin fluorescence under the amber LED excitation. Each LED was sequentially turned on and off and corresponding fluorescence signals were measured by the highly sensitive silicon photomultiplier. Future work will include implementing additional wavelengths of excitation LEDs to broaden the range of excitation wavelength and a monochromator to scan the wide range of fluorescence emission wavelength.

\section{Authors' contributions}

YHS and JWC designed the research and experiments. MTGW cultured the sample and supported characterization. YHS and MTGW analyzed the data. YSH wrote the paper. JWC supervised the research and preparation of the manuscript. All authors read and approved the final manuscript.

\section{Author details}

1 School of Electrical Engineering and Computer Science, Louisiana State University, Baton Rouge, LA, USA. ${ }^{2}$ Aquatic Germplasm and Genetic Resources
Center, School of Renewable Natural Resources, Louisiana State University Agricultural Center, Baton Rouge, LA, USA. ${ }^{3}$ Center for Advanced Microstructures and Devices, Louisiana State University, Baton Rouge, LA, USA.

\section{Acknowledgements}

Not applicable.

\section{Competing interests}

The authors declare that they have no competing interests.

\section{Availability of data and materials}

The datasets supporting the conclusions of this article are included within the article.

\section{Funding}

This research was supported in part by National Science Foundation EPSCOR and Louisiana Board of Regents, Contract LEQSF-EPS(2014)-PFUND-347.

\section{Publisher's Note}

Springer Nature remains neutral with regard to jurisdictional claims in published maps and institutional affiliations.

Received: 1 October 2018 Accepted: 15 December 2018

Published online: 18 December 2018

\section{References}

1. Spatharis S, Tsirtsis G (2010) Ecological quality scales based on phytoplankton for the implementation of water framework directive in the Eastern Mediterranean. Ecol Ind 10:840-847

2. Caron DA, Countway PD, Jones AC, Kim DY, Schnetzer A (2012) Marine protistan diversity. Ann Rev Mar Sci 4:467-493

3. Liu S, Yao P, Yu Z, Li D, Deng C, Zhen Y (2014) HPLC pigment profiles of 31 harmful algal bloom species isolated from the coastal sea areas of China. J Ocean Univ China 13:941-950

4. Hyka P, Lickova S, Pribyl P, Melzoch K, Kovar K (2013) Flow cytometry for the development of biotechnological processes with microalgae. Biotechnol Adv 31:2-16

5. Beutler M, Wiltshire KH, Meyer B, Moldaenke C, Lüring C, Meyerhöfer M, Hansen U-P, Dau H (2002) A fluorometric method for the differentiation of algal populations in vivo and in situ. Photosynth Res 72:39-53

6. Shin Y-H, Barnett JZ, Gutierrez-Wing MT, Rusch KA, Choi J-W (2015) A portable fluorescent sensor for on-site detection of microalgae. Microelectron Eng 144:6-11

7. Shin Y-H, Barnett JZ, Gutierrez-Wing MT, Rusch KA, Choi J-W (2018) A hand-held fluorescent sensor platform for selectively estimating green algae and cyanobacteria biomass. Sensors Actuators B Chem 262:938-946 
8. Lohrenz SE, Weidemann AD, Tuel M (2003) Phytoplankton spectral absorption as influenced by community size structure and pigment composition. J Plankton Res 25:35-61

9. Baker NR (2008) Chlorophyll fluorescence: a probe of photosynthesis in vivo. Annu Rev Plant Biol 59:89-113

10. Brient L, Lengronne M, Bertrand E, Rolland D, Sipel A, Steinmann D, Baudin I, Legeas M, Le Rouzic B, Bormans M (2008) A phycocyanin probe as a tool for monitoring cyanobacteria in freshwater bodies. J Environ Monit 10:248-255
11. WHO (2003) Guidelines for safe recreational water environments, Coastal and Fresh Waters. World Health Organization, Geneva, p 1

12. Su Y, Chen F, Liu Z (2015) Comparison of optical properties of chromophoric dissolved organic matter (CDOM) in alpine lakes above or below the tree line: insights into sources of CDOM. Photochem Photobiol Sci $14: 1047-1062$

\section{Submit your manuscript to a SpringerOpen ${ }^{\circ}$ journal and benefit from:}

- Convenient online submission

- Rigorous peer review

- Open access: articles freely available online

- High visibility within the field

- Retaining the copyright to your article

Submit your next manuscript at $\boldsymbol{\nabla}$ springeropen.com 